\title{
Adaptive Power Allocation in Spatially Correlated Dual-Hop MIMO Relay Channels
}

\author{
Ah-Young Kim \\ Seoul National University \\ Kwanak P. O. Box 34, \\ Seoul 151-600 Korea \\ $+82-2-880-7281$ \\ ahyoung@ttl.snu.ac.kr
}

\author{
Hee-Nam Cho \\ Seoul National University \\ Kwanak P. O. Box 34, \\ Seoul 151-600 Korea \\ $+82-2-880-7281$ \\ hncho@ttl.snu.ac.kr
}

\author{
Yong-Hwan Lee \\ Seoul National University \\ Kwanak P. O. Box 34, \\ Seoul 151-600 Korea \\ $+82-2-880-8413$ \\ ylee@snu.ac.kr
}

\begin{abstract}
This paper considers power allocation in a spatially correlated dual-hop multiple-input multiple-output (MIMO) relay channel. In a dual-hop MIMO relay channel, it is desirable to balance the capacity of each hop channel by dynamically allocating the transmit power according to the channel condition. In fact, the end-to-end capacity of a dual-hop relay channel can be maximized by means of max-min optimization with constraint on total power. This paper considers the improvement of the end-toend capacity by allocating the transmit power according to the average channel gain and the spatial correlation between the dualhop MIMO relay channels. Simulation results show that the proposed scheme provides significant performance improvement over the power allocation based on the average signal-to-noise ratio (SNR).
\end{abstract}

\section{Categories and Subject Descriptors}

B.8.2 [Performance and reliability]: Performance Analysis and Design Aids - relay channels, power allocation.

\section{General Terms}

Design, Performance, Experimentation

\section{Keywords}

Channel correlation, dual-hop MIMO relay channels, power allocation

\section{INTRODUCTION}

The use of relays with multiple-input multiple-output (MIMO), so-called MIMO relays, has actively been considered in wireless networks due to its potential for the enhancement of capacity as well as cell coverage [1]-[5]. Much effort has been concentrated on resource allocation since the end-to-end capacity of a MIMO relay channel can be increased by adaptively allocating the resource according to the channel condition [6], [7].

Permission to make digital or hard copies of all or part of this work for personal or classroom use is granted without fee provided that copies are not made or distributed for profit or commercial advantage and that copies bear this notice and the full citation on the first page. To copy otherwise, or republish, to post on servers or to redistribute to lists, requires prior specific permission and/or a fee.

IWCMC'09, June 21-24, 2009, Leipzig, Germany.

Copyright $($ C 2009 ACM 978-1-60558-569-7/09/06 ..\$5.00.
In a spatially uncorrelated dual-hop MIMO relay channel, the end-to-end capacity can be improved by adaptively allocating the transmit power according to the average signal-to-noise ratio (SNR) of each hop channel [8]. However, the average SNR-based power allocation scheme may experience performance degradation in correlated dual-hop MIMO relay channels [9]-[12].

This is mainly because the channel gain is highly concentrated on a few eigen-dimensions of the correlation matrix as the channel correlation increases [13], [14], reducing the effective degree of freedom. In this case, it may be sufficient to allocate the transmit power to a subspace spanned by eigenvectors corresponding to dominant eigenvalues. It is of great concern how to allocate the transmit power based on the characteristics of the channel correlation in dual-hop MIMO relay channel.

In this paper, we consider power allocation to the source and relay in correlated dual-hop MIMO relay channel. We first derive the capacity of a correlated single-hop MIMO channel, and then sufficient condition to maximize the end-to-end capacity of a correlated dual-hop MIMO relay channel by means of a max-min technique. The proposed scheme adaptively allocates the transmit power to balance the capacity between the dual-hop MIMO relay channels according to the average channel gain and the spatial channel correlation. The performance of the proposed scheme is verified by computer simulation.

The remainder of this paper is organized as follows. Section 2 describes correlated dual-hop MIMO relay channels in consideration. Section 3 proposes a correlation-based power allocation strategy to maximize the end-to-end capacity in a correlated dual-hop MIMO relay channel. Section 4 verifies the analytic results by computer simulation. Finally, conclusions are given in Section 5.

\section{SYSTEM MODEL}

We consider a single-user dual-hop MIMO relay channel as shown in Figure 1, where the source transmits the signal using two antennas to the relay through the first hop channel, the relay receives the signal using two antennas and retransmits it through the second hop channel, and the destination receives it using two antennas. We assume that the first and the second hop channel equally share the available channel bandwidth, and that the total sum power of the source and relay is $P$. We also assume that the direct link between the source and the destination is not available due to large path loss. 


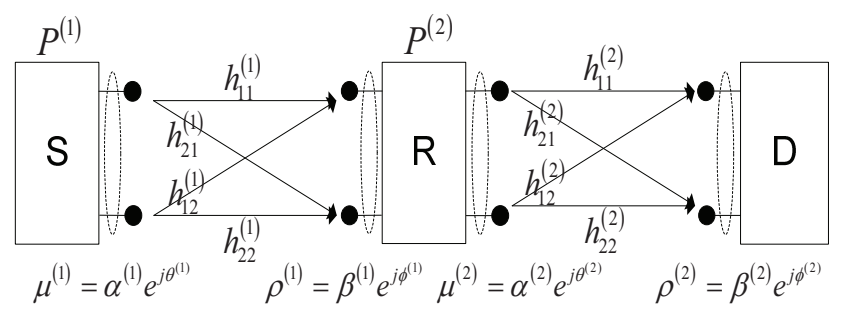

Figure 1. Correlated dual-hop MIMO relay channel.

Let $\mathbf{x}^{(i)}$ and $\mathbf{H}^{(i)}$ be the transmit signal vector and the channel matrix of the $i$-th hop channel, respectively. Then, the received signal through the $i$-th hop channel can be represented as

$$
\mathbf{y}^{(i)}=\sqrt{\eta^{(i)}} \mathbf{H}^{(i)} \mathbf{x}^{(i)}+\mathbf{n}^{(i)}
$$

where $\mathbf{n}^{(i)}$ denotes zero-mean additive white Gaussian noise (AWGN) with variance $N_{0}$ and $\eta^{(i)}\left(\triangleq P^{(i)} d^{(i)^{-\xi}}\right)$ denotes the large-scale fading coefficient of the $i$-th hop channel. Here, $P^{(i)}$, $d^{(i)}$ and $\xi$ denote the transmit power, the propagation distance and the path loss exponent of the $i$-th hop channel, respectively.

The corresponding $2 \times 2$ channel matrix $\mathbf{H}^{(i)}$ of the $i$-th hop channel can be represented as

$$
\mathbf{H}^{(i)}=\left[\begin{array}{ll}
h_{11}^{(i)} & h_{12}^{(i)} \\
h_{21}^{(i)} & h_{22}^{(i)}
\end{array}\right]
$$

where the $(m, n)$-th element $h_{m n}^{(i)}$ represents the channel gain from the $n$-th transmit antenna to the $m$-th receive antenna through the $i$-th hop channel. When the MIMO channel is spatially correlated, the channel matrix $\mathbf{H}^{(i)}$ can be generated using an independent and identically distributed (i.i.d.) Rayleigh channel matrix $\tilde{\mathbf{H}}^{(i)}$ by [10]

$$
\operatorname{vec}\left(\mathbf{H}^{(i)}\right)=\mathbf{R}^{(i) / 2} \operatorname{vec}\left(\tilde{\mathbf{H}}^{(i)}\right)
$$

where $\operatorname{vec}\left(\mathbf{H}^{(i)}\right)$ denotes an operator that stacks matrix $\mathbf{H}^{(i)}$ into a vector columnwise and $\mathbf{R}^{(i) / 2}$ denotes the square root of the channel correlation matrix $\mathbf{R}^{(i)}$ which is Hermitian and positive definite. Then, the channel correlation matrix $\mathbf{R}^{(i)}$ can be represented as [15]

$$
\begin{aligned}
\mathbf{R}^{(i)} & =E\left\{\operatorname{vec}\left(\mathbf{H}^{(i)}\right) \operatorname{vec}\left(\mathbf{H}^{(i)}\right)^{*}\right\} \\
& =\left[\begin{array}{cccc}
1 & \rho^{(i)} & \mu^{(i)} & \mu^{(i)} \rho^{(i)} \\
\rho^{(i)^{*}} & 1 & \mu^{(i)} \rho^{(i)^{*}} & \mu^{(i)} \\
\mu^{(i)^{*}} & \mu^{(i)^{*}} \rho^{(i)} & 1 & \rho^{(i)} \\
\mu^{(i)^{*}} \rho^{(i)^{*}} & \mu^{(i)^{*}} & \rho^{(i)^{*}} & 1
\end{array}\right] \\
& =\mathbf{R}_{t}^{(i)} \otimes \mathbf{R}_{r}^{(i)}
\end{aligned}
$$

where $\otimes$ denotes Kronecker product. Here, $\mathbf{R}_{t}{ }^{(i)}$ and $\mathbf{R}_{r}{ }^{(i)}$ are respectively the transmit and receive correlation matrix defined by

$$
\mathbf{R}_{t}^{(i)}=\left[\begin{array}{cc}
1 & \mu^{(i)} \\
\mu^{(i)^{*}} & 1
\end{array}\right] \text { and } \mathbf{R}_{r}^{(i)}=\left[\begin{array}{cc}
1 & \rho^{(i)} \\
\rho^{(i)^{*}} & 1
\end{array}\right]
$$

where the superscript $*$ denotes complex conjugate transpose, $\mu^{(i)}\left(\triangleq \alpha^{(i)} e^{j \theta^{(i)}}\right)$ and $\rho^{(i)}\left(\triangleq \beta^{(i)} e^{j \phi^{(i)}}\right)$ are the transmit and receive correlation coefficient between the adjacent antennas, respectively. Here, $\alpha^{(i)}\left(0 \leq \alpha^{(i)} \leq 1\right)$ and $\theta^{(i)}\left(0 \leq \theta^{(i)}<2 \pi\right)$ denote the amplitude and the phase of $\mu^{(i)}$, and $\beta^{(i)}$ $\left(0 \leq \beta^{(i)} \leq 1\right)$ and $\phi^{(i)} \quad\left(0 \leq \phi^{(i)}<2 \pi\right)$ denote the amplitude and the phase of $\rho^{(i)}$, respectively.

\section{PROPOSED POWER ALLOCATION SCHEME}

We consider correlation-based power allocation in a dual-hop MIMO relay channel. We first briefly review a conventional power allocation scheme for easy description of the proposed scheme. In an uncorrelated dual-hop MIMO relay channel, the transmit power in the first and the second hop channel can be determined based on the average channel gain as [8]

$$
\begin{aligned}
\left(P_{\mathrm{SNR}}^{(1)}, P_{\mathrm{SNR}}^{(2)}\right)= & \left(\frac{\sqrt[3]{d^{(2)^{-\xi}} E^{2}\left\{\sqrt{\lambda^{(2)}}\right\}}}{\sqrt[3]{d^{(1)^{-\xi}} E^{2}\left\{\sqrt{\lambda^{(1)}}\right\}}+\sqrt[3]{d^{(2)^{-\xi}} E^{2}\left\{\sqrt{\lambda^{(2)}}\right\}}} P,\right. \\
& \left.\frac{\sqrt[3]{d^{(1)^{-\xi}} E^{2}\left\{\sqrt{\lambda^{(1)}}\right\}}}{\sqrt[3]{d^{(1)^{-\xi}} E^{2}\left\{\sqrt{\lambda^{(1)}}\right\}}+\sqrt[3]{d^{(2)^{-\xi}} E^{2}\left\{\sqrt{\lambda^{(2)}}\right\}}} P\right)
\end{aligned}
$$

where $E\left\{\sqrt{\lambda^{(i)}}\right\}=\Gamma\left(M^{(i)}+1 / 2\right) / \Gamma\left(M^{(i)}\right)$. Here, $M^{(i)}$ denotes the number of transmit antennas in the $i$-th hop channel and $\Gamma(x)=\int_{0}^{\infty} z^{x-1} e^{-z} d z$ for $x>0$. It can be seen from (6) that $P_{\mathrm{SNR}}^{(i)}$ is inversely proportional to the average channel gain of the $i$-th hop channel. However, the presence of channel correlation reduces the effective degrees of freedom, so-called rank deficiency, causing the reduction of the capacity of MIMO channel. That is, as the channel correlation of the $i$-th hop channel increases, the capacity decreases due to the rank deficiency. It makes the capacity between the dual-hop MIMO relay channels imbalanced.

To alleviate this problem, we first derive the capacity of a correlated single-hop MIMO channel and then determine the transmit power to maximize the end-to-end capacity of a correlated dual-hop MIMO relay channel by means of a max-min technique. The end-to-end capacity of a dual-hop MIMO relay channel is determined by the minimum capacity of hop channels, i.e., $\quad C=\min \left(C^{(1)}\left(P^{(1)}\right), C^{(2)}\left(P^{(2)}\right)\right) \quad$ [6]. It is desirable to distribute the total power $P$ to maximize the minimum capacity 
of each hop channel. Thus, the optimum power allocation problem for a dual-hop MIMO relay channel can be formulated

$$
C=\max _{\left(P^{(1)}, P^{(2)}\right)} \min \left\{C^{(1)}\left(P^{(1)}\right), C^{(2)}\left(P^{(2)}\right)\right\}
$$

where $P^{(1)}+P^{(2)}=P$. It can be shown by means of a max-min technique [16] that the end-to-end capacity is maximized by making

$$
C^{(1)}\left(P^{(1)}\right)=C^{(2)}\left(P^{(2)}\right)
$$

In a correlated dual-hop MIMO relay channel, the capacity of the $i$-th hop channel can be represented as

$$
C^{(i)}=E\left\{\log _{2} \operatorname{det}\left(\mathbf{I}_{2}+\frac{\gamma^{(i)}}{2} \mathbf{H}^{(i)} \mathbf{H}^{(i)^{*}}\right)\right\}
$$

where $\gamma^{(i)}$ denotes the average SNR. From Jensen's inequality, it can be shown that [17]

$$
\begin{aligned}
& C_{i} \leq \log _{2} E\left\{\operatorname{det}\left[\begin{array}{cc}
1+\gamma^{(i)}\left(h_{11}^{(i)} h_{11}^{(i)^{*}}+h_{12}^{(i)} h_{12}^{(i)^{*}}\right) / 2 & \gamma^{(i)}\left(h_{11}^{(i)} h_{21}^{(i)^{*}}+h_{12}^{(i)} h_{22}^{(i)^{*}}\right) / 2 \\
\gamma^{(i)}\left(h_{21}^{(i)} h_{11}^{(i)}+h_{22}^{(i)} h_{12}^{(i)^{*}}\right) / 2 & 1+\gamma^{(i)}\left(h_{21}^{(i)} h_{21}^{(i)^{*}}+h_{22}^{(i)} h_{22}^{(i)^{*}}\right) / 2
\end{array}\right]\right\} \\
& =\log _{2} E\left\{\begin{array}{l}
1+\frac{\gamma^{(i)}}{2}\left(\left|h_{11}^{(i)}\right|^{2}+\left|h_{12}^{(i)}\right|^{2}+\left|h_{21}^{(i)}\right|^{2}+\left|h_{22}^{(i)}\right|^{2}\right) \\
+\frac{\gamma^{(i)^{2}}}{4}\left(\begin{array}{l}
\left|h_{11}^{(i)}\right|^{2}\left|h_{22}^{(i)}\right|^{2}+\left|h_{12}^{(i)}\right|^{2}\left|h_{21}^{(i)}\right|^{2}- \\
h_{11}^{(i)} h_{12}^{(i)^{*}} h_{21}^{(i) *} h_{22}^{(i)}-h_{11}^{(i)^{*}} h_{12}^{(i)} h_{21}^{(i)} h_{22}^{(i)^{*}}
\end{array}\right)
\end{array}\right\} \\
& =\log _{2}\left\{1+2 \gamma^{(i)}+\frac{\gamma^{(i)^{2}}}{2}\left(1+\left|\rho^{(i)} \mu^{(i)}\right|^{2}-\left|\rho^{(i)}\right|^{2}-\left|\mu^{(i)}\right|^{2}\right)\right\} \\
& =\log _{2}\left\{1+2 \gamma^{(i)}+\frac{\gamma^{(i)^{2}}}{2}\left(1-\alpha^{(i)^{2}}\right)\left(1-\beta^{(i)^{2}}\right)\right\} \text {. }
\end{aligned}
$$

Thus, we have

$$
C^{(i)} \leq \log _{2}\left(1+2 \gamma^{(i)}+\frac{\gamma^{(i)^{2}}}{2} K^{(i)}\right)
$$

where $K^{(i)}=\left(1-\alpha^{(i)^{2}}\right)\left(1-\beta^{(i)^{2}}\right)$. It can be seen from (11) that the capacity of a single-hop MIMO channels decreases as $\alpha^{(i)}$ and $\beta^{(i)}$ increase.

Thus, it can be seen that the condition for (8) is given by

$$
2 \gamma^{(1)}+\frac{\gamma^{(1)^{2}}}{2} K^{(1)}=2 \gamma^{(2)}+\frac{\gamma^{(2)^{2}}}{2} K^{(2)} .
$$

Since $\gamma^{(i)}=P^{(i)} d^{(i)^{-\xi}} / N_{0}$ and $P^{(1)}+P^{(2)}=P \quad, \quad$ (12) can be rewritten as

$$
\begin{aligned}
& 2 \frac{P^{(1)} d^{(1)^{-\xi}}}{N_{0}}+\frac{P^{(1)^{2}} d^{(1)^{-2 \xi}}}{2 N_{0}^{2}} K^{(1)} \\
& =2 \frac{\left(P-P^{(1)}\right) d^{(2)^{-\xi}}}{N_{0}}+\frac{\left(P-P^{(1)}\right)^{2} d^{(2)^{-2 \xi}}}{2 N_{0}^{2}} K^{(2)} .
\end{aligned}
$$

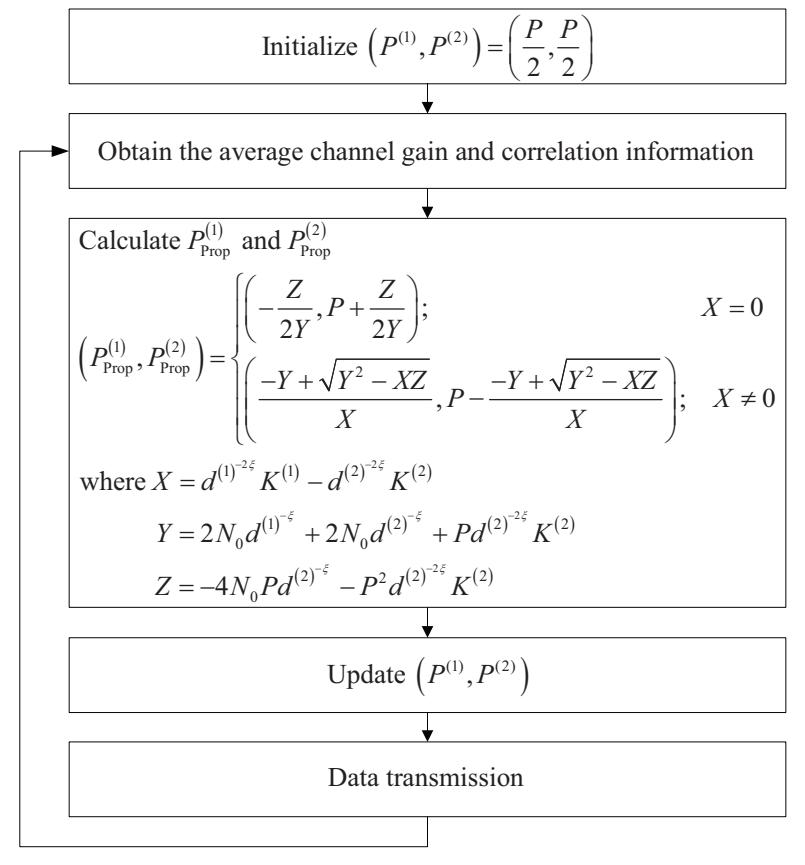

Figure 2. Procedure of the proposed scheme.

This implies that the end-to-end capacity can be maximized by allocating the power as

$$
\begin{aligned}
& \left(P_{\text {Prop }}^{(1)}, P_{\text {Prop }}^{(2)}\right) \\
& = \begin{cases}\left(-\frac{Z}{2 Y}, P+\frac{Z}{2 Y}\right) ; & X=0 \\
\left(\frac{-Y+\sqrt{Y^{2}-X Z}}{X}, P-\frac{-Y+\sqrt{Y^{2}-X Z}}{X}\right) ; & X \neq 0\end{cases}
\end{aligned}
$$

where

$$
\begin{gathered}
X=d^{(1)^{-2 \xi}} K^{(1)}-d^{(2)^{-2 \xi}} K^{(2)} \\
Y=2 N_{0} d^{(1)^{-\xi}}+2 N_{0} d^{(2)^{-\xi}}+P d^{(2)^{-2 \xi}} K^{(2)} \\
Z=-4 N_{0} P d^{(2)^{-\xi}}-P^{2} d^{(2)^{-2 \xi}} K^{(2)} .
\end{gathered}
$$

It can be seen that the proposed power allocation highly depends on the correlation amplitude $\alpha^{(i)}$ and $\beta^{(i)}$ of the correlation coefficient as well as the average channel gain $d^{(i)^{-\zeta}}$. In fact, $P_{\text {Prop }}^{(i)}$ is proportional to the correlation amplitude of the $i$-th hop channel and inversely proportional to the average channel gain. Thus, as the correlation amplitude of the first hop channel increases, $P_{\text {Prop }}^{(1)}$ needs to be increased to balance the capacity between the first and the second hop channel, and vice versa.

As illustrated in Figure 2, the overall procedure of the BS with the proposed scheme can be summarized as follows:

- Step 1 : Initialize the transmit power with equal power allocation.

- Step 2 : The relay and the user estimate the average channel gain and correlation information using common pilot 
signal, and report them to the BS through an uplink control channel.

- Step 3 : The BS determines the transmit power $P_{\text {Prop }}^{(1)}$ and $P_{\text {Prop }}^{(2)}$ by (14).

- Step 4 : Update the transmit power $\left(P^{(1)}, P^{(2)}\right)$.

- Step 5 : The BS transmits the data to the relay through the first hop channel and the relay re-transmits it to the user through the second hop channel.

\section{SIMULATION RESULTS}

The performance of the proposed scheme is verified by computer simulation in a correlated dual-hop $2 \times 2$ MIMO relay channel. We assume that the amplitude of the transmit and receive correlation coefficients is the same in each hop channel (i.e., $\alpha^{(i)}=\beta^{(i)}$ for $\left.i=1,2\right)$. For comparison, the performance of the average SNR-based scheme is also considered, where the total power is distributed according to the average channel gain between the two hop channels. The common simulation parameters are summarized in Table 1.

Figure 3 depicts the performance of the proposed scheme in a correlated dual-hop $2 \times 2$ MIMO channel according to $\alpha^{(2)}\left(=\beta^{(2)}\right) \quad$ when $\quad \gamma_{0}=0 \quad \mathrm{~dB}, \quad d^{(1)}=d^{(2)}=0.5 \quad \mathrm{~km}, \quad$ and $\alpha^{(1)}=\beta^{(1)}=0.3$. It can be seen from Figure 3 (a) that the proposed scheme adaptively allocates the power according to $\alpha^{(2)}\left(=\beta^{(2)}\right)$. On the other hand, the average SNR-based power allocation scheme allocates constant power indifferently from $\alpha^{(2)}\left(=\beta^{(2)}\right)$. As $\alpha^{(2)}\left(=\beta^{(2)}\right)$ increases, $P_{\text {Prop }}^{(2)}$ increases to balance the capacity between the dual-hop MIMO relay channels. It can also be seen from Figure 3 (b) that the end-to-end capacity of the two schemes gradually decreases as $\alpha^{(2)}\left(=\beta^{(2)}\right)$ increases. This is mainly because the capacity of the second hop channel decreases due to the rank deficiency. However, the proposed scheme allocates the

Table 1. Simulation parameters

\begin{tabular}{|c|c|}
\hline System configuration & $\begin{array}{c}\text { Dual-hop } 2 \times 2 \text { MIMO relay } \\
\text { channels }\end{array}$ \\
\hline Cell radius & $1 \mathrm{~km}$ \\
\hline Relay protocol & $\begin{array}{c}\text { Regenerative relay with } \\
\text { decode-and-forward mode }\end{array}$ \\
\hline Sum power & 1 \\
\hline Fading channel & Spatially correlated Rayleigh fading \\
\hline Path loss exponent & 4 \\
\hline Link adaptation & $\begin{array}{c}\text { Ideal (i.e., using the Shannon's } \\
\text { capacity formula) }\end{array}$ \\
\hline
\end{tabular}

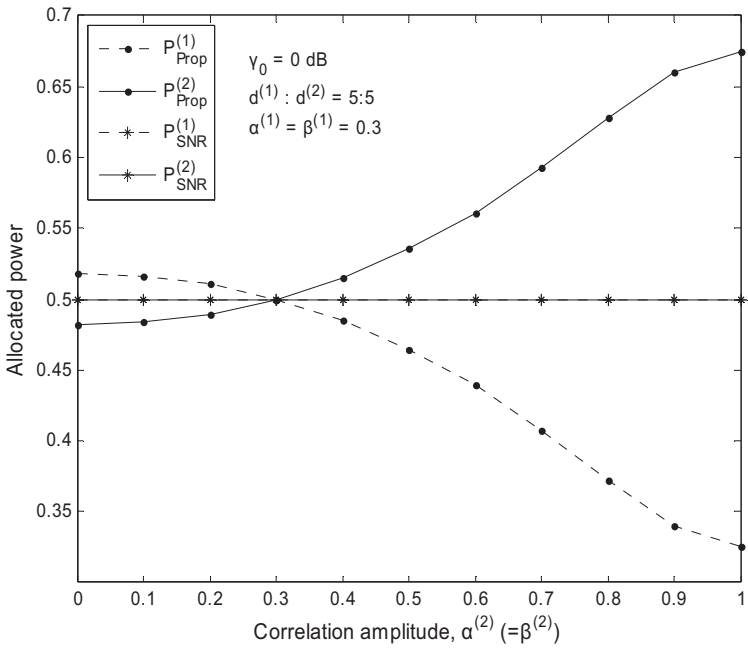

(a) Allocated power of the proposed scheme.

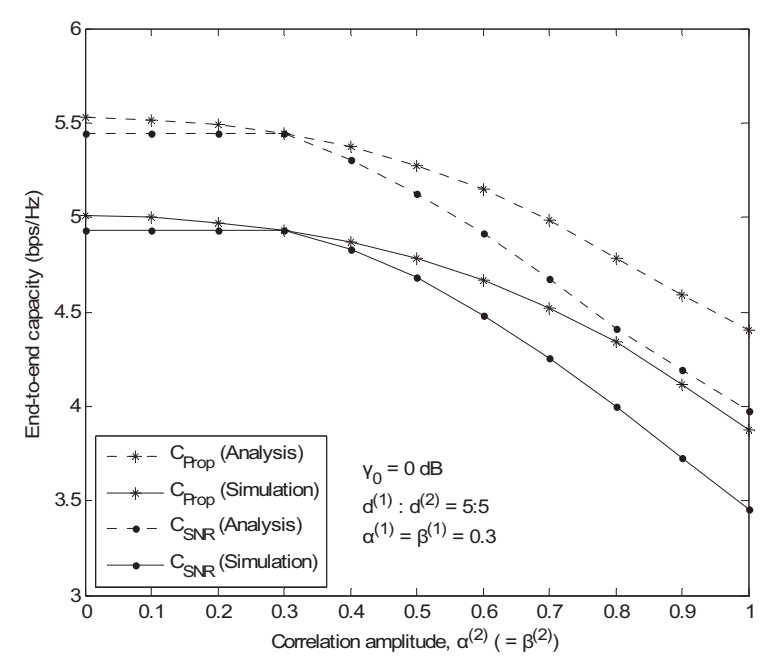

(b) End-to-end capacity of the proposed scheme.

Figure 1. End-to-end capacity of the proposed scheme according to $\alpha^{(2)}\left(=\beta^{(2)}\right)$ when $\gamma_{0}=0 \mathrm{~dB}$, $d^{(1)}=d^{(2)}=0.5$, and $\alpha^{(1)}=\beta^{(1)}=0.3$.

power in consideration of the rank deficiency, yielding less reduction of the capacity. As $\alpha^{(2)}\left(=\beta^{(2)}\right)$ increases, the performance difference between the two power allocation schemes increases. Notice that when $\alpha^{(2)}=\beta^{(2)}=0.3$, both the schemes has the same end-to-end capacity since they have the same power allocation when $\alpha^{(1)}=\beta^{(1)}=\alpha^{(2)}=\beta^{(2)}$ and $d^{(1)}=d^{(2)}$. Notice also that the analytic results show some discrepancy from the simulation results, which is due to the use of an upper bound with Jensen's inequality, but it is still useful for the estimation of the performance.

Figure 4 depicts the end-to-end capacity of a correlated dual-hop MIMO relay channels according to the average SNR $\gamma_{0}$ with the 


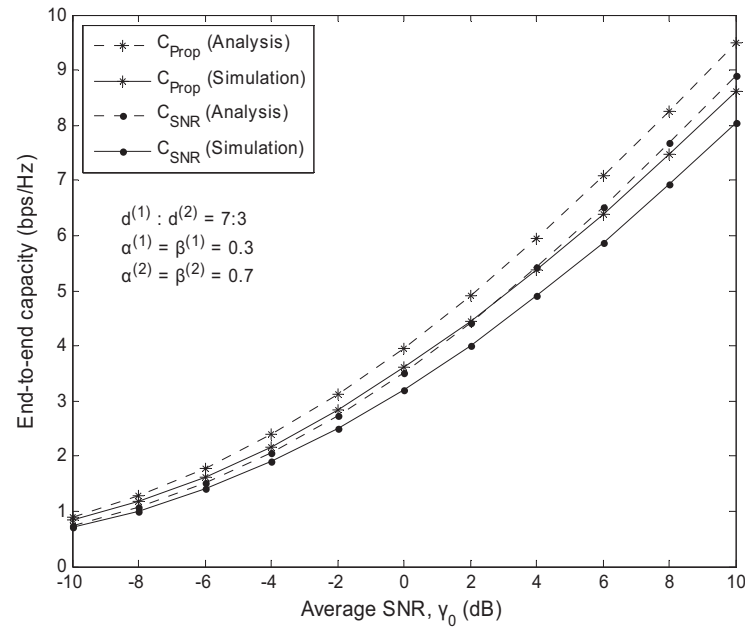

Figure 4. End-to-end capacity of the proposed scheme according to $\gamma_{0}$ when $d^{(1)}: d^{(2)}=7: 3, \alpha^{(1)}=\beta^{(1)}=0.3$, and $\alpha^{(2)}=\beta^{(2)}=0.7$.

use of the proposed scheme when $d^{(1)}=0.7 \mathrm{~km}, d^{(2)}=0.3 \mathrm{~km}$, $\alpha^{(1)}=\beta^{(1)}=0.3$, and $\alpha^{(2)}=\beta^{(2)}=0.7$. It can be seen that the proposed scheme outperforms the conventional SNR-based power allocation. The proposed scheme provides a spectral efficiency enhancement of $0.122 \mathrm{bps} / \mathrm{Hz}$ (or $17.2 \%$ improvement) and 0.56 bps $/ \mathrm{Hz}$ (or $7.0 \%$ improvement) when $\gamma_{0}=-10 \mathrm{~dB}$ and $\gamma_{0}=10$ $\mathrm{dB}$, respectively. This means that compared to the conventional scheme, the proposed scheme is much effective in low SNR environments such as coverage hole or cell boundary.

\section{CONCLUSION}

We have considered the allocation of transmit power in correlated dual-hop MIMO relay channels. The proposed scheme allocates the power to balance the capacity of each hop channel by considering the average channel gain and the correlation amplitude between the dual-hop MIMO relay channels. Numerical results show that the proposed scheme can provide noticeable performance improvement over the average SNRbased one in spatially correlated environments. The performance improvement is noticeable especially in low SNR environments.

\section{ACKNOWLEDGMENT}

This work was supported by the Korea Science and Engineering Foundation (KOSEF) grant funded by the Korea government (MEST) (No. R01-2008-000-21098-0)

\section{REFERENCES}

[1] T. M. Cover and A. A. El Gamel, "Capacity theorems for the relay channel," IEEE Trans. Inf. Theory, vol. 25, no. 5, pp. 572-584, Sept. 1979.

[2] R. Pabst, B. H. Walke, D. C. Schultz, P. Herhold, H. Yanikomeroglu, S. Mukherjeee, H. Viswanathan, M. Lott, W. Zirwas, M. Dohler, H. Aghvami, D. D. Falconer, and G. P.
Fettweis, "Relay-based deployment concepts for wireless and mobile broadband radio," IEEE Commun. Mag., vol. 42, no. 9, pp. 80-89, Sept. 2004.

[3] D. Soldani and S. Dixit, "Wireless relays for broadband access”, IEEE Commun. Mag., vol. 46, no. 3, pp. 58-66, Mar. 2008

[4] B. Wang, J. Zhang, and A. Høst-Madson, "On the capacity of MIMO relay channels," IEEE Trans. Inf. Theory, vol. 51, no. 1, pp. 29-43, Jan. 2005.

[5] H. Bölcskei, R. U. Nabar, Ö. Oyman, and A. J. Paulraj, "Capacity scaling laws in MIMO relay networks," IEEE Trans. Wireless Commun., vol. 5, no. 6, June 2006.

[6] A. Reznik, S. R. Kulkarni, and S. Verdú, "Degraded Gaussian multirelay channel: capacity and optimal power allocation," IEEE Trans. Inf. Theory, vol. 50, no. 12, pp. 3037-3046, Dec. 2004.

[7] Y. Zhao, R. Adve, and T. J. Lim, "Improving amplify-andforward relay networks: optimal power allocation versus selection", IEEE Trans. Wireless Commun., vol. 6, no. 8, pp. 3114-3123, Aug. 2007.

[8] M. Dohler, A. Gkelias, and H. Aghvami, "2-hop distributed MIMO communication system," IEEE Electron. Lett., vol. 39, no. 18, pp. 1350-1351, Sept. 2003.

[9] D. S. Shiu, G. J. Foschini, M. J. Gans, and J. M. Kahn, "Fading correlation and its effect on the capacity of multielement antenna systems," IEEE Trans. Commun, vol. 48, no. 3, pp. 502-513, Mar. 2000.

[10] A. M. Tulino, A. Lozano, and S. Verdú, "Impact of antenna correlation on the capacity of multiantenna channels," IEEE Trans. Inf. Theory, vol. 51, no. 7, pp. 2491-2509, July 2005.

[11] H. Shin and J. H. Lee, "Capacity of multiple-antenna fading channels: Spatial fading correlation, double scattering and keyhole," IEEE Trans. Inf. Theory, vol. 49, no. 10, pp. 2636-2647, Oct. 2003.

[12] J. P. Kermoal, L. Schumacher, K. I. Pedersen, P. E. Mogensen, and F. Frederiksen, "A stochastic MIMO radio channel model with experimental validation," IEEE J. Sel. Areas Commun., vol. 20, no. 6, pp. 1211-1226, Aug. 2002.

[13] M. T. Ivrlač, W. Utschick, and J. A. Nossek, "Fading correlations in wireless MIMO communication systems," IEEE J. Sel. Areas Commun., vol. 21, no. 5, June 2003.

[14] Chuah, J. M. Kahn, and D. Tse, "Capacity of multi-antenna array systems in indoor wireless environment," in Proc. IEEE Globecom'98, vol. 4, pp. 1894-1899, Nov. 1998.

[15] K. Yu, M. Bengtsson, B. Ottersten, D. McNamara, P. Karlsson, and M. Beach, "Second order statistics of NLOS indoor MIMO channels based on $5.2 \mathrm{GHz}$ measurements," in Proc. IEEE Globecom'01, vol. 1, pp. 156-160, Nov. 2001.

[16] J.-W. Lee and Y.-H. Lee, "Achievable sum-rate analysis of correlated two-antenna MIMO uplink channels," submitted to Int. J. Commun. Syst., Mar. 2008.

[17] V. Poor, An Introduction to Signal Detection and Estimation, Springer, 1994 